\title{
Advances in Singlet Fission Chromophore Design Enabled by Vibrational Spectroscopies
}

\author{
Supplementary Information \\ Kajari Bera ${ }^{\dagger}$ Siu Yi Kwang ${ }^{\dagger}$ Renee R. Frontiera* \\ Department of Chemistry, University of Minnesota \\ Minneapolis, MN 55455 \\ †These authors contributed equally to the manuscript
}

*To whom correspondence should be addressed: rrf@umn.edu, 612-624-2501 
In Figure 1 of the main text, we listed various spectroscopic techniques that have been applied to studying each corresponding step of the singlet fission process. Here, we list the corresponding references from year 2000 onwards. Transient absorption is excluded because it is the most widely used technique (210 studies since year 2000). To the best of our abilities, the references are accurate as of May 2020.

Here are a list of acronyms and alternative names for the techniques used:

\begin{tabular}{|l|l|}
\hline Acronym & Technique name(s) \\
\hline TR-PL & $\begin{array}{l}\text { Time resolved photoluminescence, time resolved fluorescence, time resolved } \\
\text { emission, time-correlated single-photon counting }\end{array}$ \\
\hline 2DES & Two dimensional electronic spectroscopy \\
\hline TR-2PPE & Time resolved 2 photon photoemission spectroscopy \\
\hline TR-EPR & Time resolved electron paramagnetic/spin resonance \\
\hline TRIR & Time resolved infrared spectroscopy, transient mid-infrared spectroscopy \\
\hline ISRS & Impulsive stimulated Raman spectroscopy \\
\hline RR & Resonance Raman spectroscopy \\
\hline FSRS & Femtosecond stimulated Raman spectroscopy, ultrafast Raman loss spectroscopy \\
\hline TAM & $\begin{array}{l}\text { Transient absorption microscopy, pump-probe microscopy, 2D white light } \\
\text { microscopy }\end{array}$ \\
\hline SO-FSRS & Spatially-offset femtosecond stimulated Raman spectroscopy \\
\hline
\end{tabular}

\section{Step 1}

\begin{tabular}{|l|l|}
\hline Technique & References \\
\hline TR-PL & $1-123$ \\
\hline 2DES & $124-126$ \\
\hline TR-2PPE & $127-132$ \\
\hline TR-EPR & $8,9,11,19,107,121,133-143$ \\
\hline TRIR & $104,109,144-146$ \\
\hline ISRS & $86,91,147-149$ \\
\hline RR & $86,124,125,150,151$ \\
\hline FSRS & $152-155$ \\
\hline
\end{tabular}

\section{Step 2}

\begin{tabular}{|l|l|}
\hline Technique & References \\
\hline 2DES & 125,126 \\
\hline TRIR & $104,144-146$ \\
\hline FSRS & 153,155 \\
\hline
\end{tabular}


Step 3

\begin{tabular}{|l|l|}
\hline Technique & References \\
\hline TR-PL & $\begin{array}{l}1-3,7,9,10,19-22,24,25,28-32,34,36-40,42,43,46,49,53,60-66,68,72,73,75,79,81,85,88,90-93,95,96,102,107-109,113- \\
\\
115,120,156,157\end{array}$ \\
\hline 2DES & $124-126$ \\
\hline TR-2PPE & $127-132$ \\
\hline TR-EPR & $8,9,11,19,107,121,133-143$ \\
\hline TRIR & $104,109,144,146$ \\
\hline RR & $124,125,150,151$ \\
\hline
\end{tabular}

Step 4

\begin{tabular}{|l|l|}
\hline Technique & References \\
\hline TR-PL & $16,31,158-167,41,44,50,59,66,105,122,123$ \\
\hline TR-2PPE & $127,129,130,132$ \\
\hline
\end{tabular}

Step 5

\begin{tabular}{|l|l|}
\hline Technique & References \\
\hline $\begin{array}{l}\text { Delayed } \\
\text { fluorescence / } \\
\text { phosphorescence }\end{array}$ & $16,13-15,17,25,32-35,39,44,47,48,52,54,59,61,69,70,71,74,78,80,82,85,89,90,101,105,106,108,111,116,156,157$, \\
\hline
\end{tabular}

\section{Step 6}

\begin{tabular}{|l|l|}
\hline Technique & References \\
\hline $\begin{array}{l}\text { Delayed } \\
\text { fluorescence } \\
\text { microscopy }\end{array}$ & 48 \\
\hline TAM & $171-176$ \\
\hline SO-FSRS & 177 \\
\hline
\end{tabular}

Others

\begin{tabular}{|l|l|}
\hline Technique & References \\
\hline $\begin{array}{l}\text { Magnetic field } \\
\text { dependence of } \\
\text { fluorescence }\end{array}$ & $32,33,49-51,53,57,75,81,82,87,90,114,118,122,135,158,161,170,178-197$ \\
\hline
\end{tabular}




\section{References}

(1) Müller, A. M.; Avlasevich, Y. S.; Müllen, K.; Bardeen, C. J. Evidence for Exciton Fission and Fusion in a Covalently Linked Tetracene Dimer. Chem. Phys. Lett. 2006, 421 (4-6), 518-522. https://doi.org/10.1016/j.cplett.2006.01.117.

(2) Müller, A. M.; Avlasevich, Y. S.; Schoeller, W. W.; Müllen, K.; Bardeen, C. J. Exciton Fission and Fusion in Bis(Tetracene) Molecules with Different Covalent Linker Structures. J. Am. Chem. Soc. 2007, 129 (46), 14240-14250. https://doi.org/10.1021/ja073173y.

(3) Burdett, J. J.; Bardeen, C. J. Quantum Beats in Crystalline Tetracene Delayed Fluorescence Due to Triplet Pair Coherences Produced by Direct Singlet Fission. J. Am. Chem. Soc. 2012, 134 (20), 8597-8607. https://doi.org/10.1021/ja301683w.

(4) Felter, K. M.; Dubey, R. K.; Grozema, F. C. Relation between Molecular Packing and Singlet Fission in Thin Films of Brominated Perylenediimides. J. Chem. Phys. 2019, 151 (9), 094301. https://doi.org/10.1063/1.5110306.

(5) Kuroda, K.; Yazaki, K.; Tanaka, Y.; Akita, M.; Sakai, H.; Hasobe, T.; Tkachenko, N. V.; Yoshizawa, M. A Pentacene-Based Nanotube Displaying Enriched Electrochemical and Photochemical Activities. Angew. Chemie - Int. Ed. 2019, 58 (4), 1115-1119. https://doi.org/10.1002/anie.201812976.

(6) Hetzer, C.; Basel, B. S.; Kopp, S. M.; Hampel, F.; White, F. J.; Clark, T.; Guldi, D. M.; Tykwinski, R. R. Chromophore Multiplication To Enable Exciton Delocalization and Triplet Diffusion Following Singlet Fission in Tetrameric Pentacene. Angew. Chemie - Int. Ed. 2019, 58 (43), 15263-15267. https://doi.org/10.1002/anie.201907221.

(7) Ryerson, J. L.; Zaykov, A.; Aguilar Suarez, L. E.; Havenith, R. W. A.; Stepp, B. R.; Dron, P. I.; Kaleta, J.; Akdag, A.; Teat, S. J.; Magnera, T. F.; et al. Structure and Photophysics of Indigoids for Singlet Fission: Cibalackrot. J. Chem. Phys. 2019, 151 (18), 184903. https://doi.org/10.1063/1.5121863.

(8) Chen, M.; Krzyaniak, M. D.; Nelson, J. N.; Bae, Y. J.; Harvey, S. M.; Schaller, R. D.; Young, R. M.; Wasielewski, M. R. Quintet-Triplet Mixing Determines the Fate of the Multiexciton State Produced by Singlet Fission in a Terrylenediimide Dimer at Room Temperature. Proc. Natl. Acad. Sci. U. S. A. 2019, 116 (17), 8178-8183. https://doi.org/10.1073/pnas.1820932116.

(9) Matsui, Y.; Kawaoka, S.; Nagashima, H.; Nakagawa, T.; Okamura, N.; Ogaki, T.; Ohta, E.; Akimoto, S.; Sato-Tomita, A.; Yagi, S.; et al. Exergonic Intramolecular Singlet Fission of an Adamantane-Linked Tetracene Dyad via Twin Quintet Multiexcitons. J. Phys. Chem. C 2019, 123 (31), 18813-18823. https://doi.org/10.1021/acs.jpcc.9b04503.

(10) Buchanan, E. A.; Kaleta, J.; Wen, J.; Lapidus, S. H.; Císařová, I.; Havlas, Z.; Johnson, J. C.; Michl, J. Molecular Packing and Singlet Fission: The Parent and Three Fluorinated 1,3Diphenylisobenzofurans. J. Phys. Chem. Lett. 2019, 10 (8), 1947-1953. https://doi.org/10.1021/acs.jpclett.8b03875.

(11) Nakamura, S.; Sakai, H.; Nagashima, H.; Kobori, Y.; Tkachenko, N. V.; Hasobe, T. Quantitative Sequential Photoenergy Conversion Process from Singlet Fission to Intermolecular Two-Electron Transfers Utilizing Tetracene Dimer. ACS Energy Lett. 2019, 4 (1), 26-31. https://doi.org/10.1021/acsenergylett.8b01964.

(12) Datko, B. D.; Grimm, R.; Walwark, D. J.; Burnside, B.; Grey, J. K. Resolving Population Dynamics and Interactions of Multiple Triplet Excitons One Molecule at a Time. J. Chem. Phys. 
2019, 151 (4), 044203. https://doi.org/10.1063/1.5099920.

(13) Aggarwal, N.; Patnaik, A. Dimeric Conformation Sensitive Electronic Excited States of Tetracene Congeners and Their Unconventional Non-Fluorescent Behaviour. J. Chem. Sci. 2019, 131 (6). https://doi.org/10.1007/s12039-019-1626-5.

(14) Bange, S.; Scherf, U.; Lupton, J. M. Absence of Singlet Fission and Carrier Multiplication in a Model Conjugated Polymer: Tracking the Triplet Population through Phosphorescence. J. Am. Chem. Soc. 2012, 134 (4), 1946-1949. https://doi.org/10.1021/ja2102289.

(15) Finton, D. M.; Wolf, E. A.; Zoutenbier, V. S.; Ward, K. A.; Biaggio, I. Routes to Singlet Exciton Fission in Rubrene Crystals and Amorphous Films. AIP Adv. 2019, 9 (9), 095027. https://doi.org/10.1063/1.5118942.

(16) Bae, Y. J.; Christensen, J. A.; Kang, G.; Zhou, J.; Young, R. M.; Wu, Y. L.; Van Duyne, R. P.; Schatz, G. C.; Wasielewski, M. R. Substituent Effects on Energetics and Crystal Morphology Modulate Singlet Fission in 9,10-Bis(Phenylethynyl)Anthracenes. J. Chem. Phys. 2019, 151 (4), 044501. https://doi.org/10.1063/1.5110411.

(17) Alagna, N.; Pérez Lustres, J. L.; Wollscheid, N.; Luo, Q.; Han, J.; Dreuw, A.; Geyer, F. L.; Brosius, V.; Bunz, U. H. F.; Buckup, T.; et al. Singlet Fission in Tetraaza-TIPS-Pentacene Oligomers: From Fs Excitation to Ms Triplet Decay via the Biexcitonic State. J. Phys. Chem. B 2019, 123 (50), 10780-10793. https://doi.org/10.1021/acs.jpcb.9b08031.

(18) Sato, K.; Katoh, R. Fluorescence Properties of B-Perylene Crystals Prepared by a Physical Vapor Transport Method under Atmospheric Pressure. Chem. Phys. Lett. 2019, 730, 312-315. https://doi.org/10.1016/j.cplett.2019.06.031.

(19) Papadopoulos, I.; Zirzlmeier, J.; Hetzer, C.; Bae, Y. J.; Krzyaniak, M. D.; Wasielewski, M. R.; Clark, T.; Tykwinski, R. R.; Guldi, D. M. Varying the Interpentacene Electronic Coupling to Tune Singlet Fission. J. Am. Chem. Soc. 2019, 141 (15), 6191-6203. https://doi.org/10.1021/jacs.8b09510.

(20) Stuart, A. N.; Tapping, P. C.; Schrefl, E.; Huang, D. M.; Kee, T. W. Controlling the Efficiency of Singlet Fission in TIPS-Pentacene/Polymer Composite Nanoparticles. J. Phys. Chem. C 2019, 123 (10), 5813-5825. https://doi.org/10.1021/acs.jpcc.8b10163.

(21) Mora-Fuentes, J. P.; Papadopoulos, I.; Thiel, D.; Álvarez-Boto, R.; Cortizo-Lacalle, D.; Clark, T.; Melle-Franco, M.; Guldi, D. M.; Mateo-Alonso, A. Singlet Fission in Pyrene-Fused Azaacene Dimers. Angew. Chemie - Int. Ed. 2020, 59 (3), 1113-1117.

https://doi.org/10.1002/anie.201911529.

(22) Korovina, N. V.; Chang, C. H.; Johnson, J. C. Spatial Separation of Triplet Excitons Drives Endothermic Singlet Fission. Nat. Chem. 2020, 12 (4), 391-398. https://doi.org/10.1038/s41557020-0422-7.

(23) Wang, L.; Bai, S.; Wu, Y.; Liu, Y.; Yao, J.; Fu, H. Revealing the Nature of Singlet Fission under the Veil of Internal Conversion. Angew. Chemie - Int. Ed. 2020, 59 (5), 2003-2007. https://doi.org/10.1002/anie.201912202.

(24) Manna, B.; Nandi, A. Exploration of Photophysics and Presence of Long Singlet Exciton Diffusion Length in Dibenz[a,h]Anthracene Nanoaggregates. J. Photochem. Photobiol. A Chem. 2020, 392, 112407. https://doi.org/10.1016/j.jphotochem.2020.112407.

(25) Jankus, V.; Snedden, E. W.; Bright, D. W.; Arac, E.; Dai, D.; Monkman, A. P. Competition between Polaron Pair Formation and Singlet Fission Observed in Amorphous Rubrene Films. 
Phys. Rev. B - Condens. Matter Mater. Phys. 2013, 87 (22).

https://doi.org/10.1103/PhysRevB.87.224202.

(26) Chen, M.; Powers-Riggs, N. E.; Coleman, A. F.; Young, R. M.; Wasielewski, M. R. Singlet Fission in Quaterrylenediimide Thin Films. J. Phys. Chem. C 2020, 124 (5), 2791-2798. https://doi.org/10.1021/acs.jpcc.9b10397.

(27) Hong, Y.; Kim, J.; Kim, W.; Kaufmann, C.; Kim, H.; Würthner, F.; Kim, D. Efficient Multiexciton State Generation in Charge-Transfer-Coupled Perylene Bisimide Dimers via Structural Control. J. Am. Chem. Soc. 2020, 142 (17), 7845-7857. https://doi.org/10.1021/jacs.0c00870.

(28) Masoomi-Godarzi, S.; Liu, M.; Tachibana, Y.; Mitchell, V. D.; Goerigk, L.; Ghiggino, K. P.; Smith, T. A.; Jones, D. J. Liquid Crystallinity as a Self-Assembly Motif for High-Efficiency, Solution-Processed, Solid-State Singlet Fission Materials. Adv. Energy Mater. 2019, 9 (31), 1901069. https://doi.org/10.1002/aenm.201901069.

(29) Mastron, J. N.; Roberts, S. T.; McAnally, R. E.; Thompson, M. E.; Bradforth, S. E. Aqueous Colloidal Acene Nanoparticles: A New Platform for Studying Singlet Fission. J. Phys. Chem. B 2013, 117 (49), 15519-15526. https://doi.org/10.1021/jp4057972.

(30) Walker, B. J.; Musser, A. J.; Beljonne, D.; Friend, R. H. Singlet Exciton Fission in Solution. Nat. Chem. 2013, 5 (12), 1019-1024. https://doi.org/10.1038/nchem.1801.

(31) Ma, L.; Zhang, K.; Kloc, C.; Sun, H.; Soci, C.; Michel-Beyerle, M. E.; Gurzadyan, G. G. Fluorescence from Rubrene Single Crystals: Interplay of Singlet Fission and Energy Trapping. Phys. Rev. B - Condens. Matter Mater. Phys. 2013, 87 (20). https://doi.org/10.1103/PhysRevB.87.201203.

(32) Piland, G. B.; Burdett, J. J.; Kurunthu, D.; Bardeen, C. J. Magnetic Field Effects on Singlet Fission and Fluorescence Decay Dynamics in Amorphous Rubrene. J. Phys. Chem. C 2013, 117 (3), 1224-1236. https://doi.org/10.1021/jp309286v.

(33) Dillon, R. J.; Piland, G. B.; Bardeen, C. J. Different Rates of Singlet Fission in Monoclinic versus Orthorhombic Crystal Forms of Diphenylhexatriene. J. Am. Chem. Soc. 2013, 135 (46), 1727817281. https://doi.org/10.1021/ja409266s.

(34) Eaton, S. W.; Shoer, L. E.; Karlen, S. D.; Dyar, S. M.; Margulies, E. A.; Veldkamp, B. S.; Ramanan, C.; Hartzler, D. A.; Savikhin, S.; Marks, T. J.; et al. Singlet Exciton Fission in Polycrystalline Thin Films of a Slip-Stacked Perylenediimide. J. Am. Chem. Soc. 2013, 135 (39), 14701-14712. https://doi.org/10.1021/ja4053174.

(35) Wen, X.; Yu, P.; Yuan, C. T.; Ma, X.; Tang, J. Singlet and Triplet Carrier Dynamics in Rubrene Single Crystal. J. Phys. Chem. C 2013, 117 (34), 17741-17747. https://doi.org/10.1021/jp404666w

(36) Guo, J.; Ohkita, H.; Benten, H.; Ito, S. Near-IR Femtosecond Transient Absorption Spectroscopy of Ultrafast Polaron and Triplet Exciton Formation in Polythiophene Films with Different Regioregularities. J. Am. Chem. Soc. 2009, 131 (46), 16869-16880.

https://doi.org/10.1021/ja906621a.

(37) Johnson, J. C.; Akdag, A.; Zamadar, M.; Chen, X.; Schwerin, A. F.; Paci, I.; Smith, M. B.; Havlas, Z.; Miller, J. R.; Ratner, M. A.; et al. Toward Designed Singlet Fission: Solution Photophysics of Two Indirectly Coupled Covalent Dimers of 1,3-Diphenylisobenzofuran. J. Phys. Chem. B 2013, 117 (16), 4680-4695. https://doi.org/10.1021/jp310979q. 
(38) Nichols, V. M.; Rodriguez, M. T.; Piland, G. B.; Tham, F.; Nesterov, V. N.; Youngblood, W. J.; Bardeen, C. J. Assessing the Potential of Peropyrene as a Singlet Fission Material: Photophysical Properties in Solution and the Solid State. J. Phys. Chem. C 2013, 117 (33), 16802-16810. https://doi.org/10.1021/jp4051116.

(39) Tayebjee, M. J. Y.; Clady, R. G. C. R.; Schmidt, T. W. The Exciton Dynamics in Tetracene Thin Films. Phys. Chem. Chem. Phys. 2013, 15 (35), 14797. https://doi.org/10.1039/c3cp52609g.

(40) Zhang, B.; Zhang, C.; Wang, R.; Tan, Z.; Liu, Y.; Guo, W.; Zhai, X.; Cao, Y.; Wang, X.; Xiao, M. Nonlinear Density Dependence of Singlet Fission Rate in Tetracene Films. J. Phys. Chem. Lett. 2014, 5 (20), 3462-3467. https://doi.org/10.1021/jz501736y.

(41) Yost, S. R.; Lee, J.; Wilson, M. W. B.; Wu, T.; McMahon, D. P.; Parkhurst, R. R.; Thompson, N. J.; Congreve, D. N.; Rao, A.; Johnson, K.; et al. A Transferable Model for Singlet-Fission Kinetics. Nat. Chem. 2014, 6 (6), 492-497. https://doi.org/10.1038/nchem.1945.

(42) Ma, L.; Tan, K. J.; Jiang, H.; Kloc, C.; Michel-Beyerle, M. E.; Gurzadyan, G. G. Excited-State Dynamics in an $\alpha$-Perylene Single Crystal: Two-Photon- and Consecutive Two-Quantum-Induced Singlet Fission. J. Phys. Chem. A 2014, 118 (5), 838-843. https://doi.org/10.1021/jp409977f.

(43) Selvakumar, S.; Sivaji, K.; Arulchakkaravarthi, A.; Sankar, S. Electron Momentum Distribution and Singlet-Singlet Annihilation in the Organic Anthracene Molecular Crystals Using Positron 2D-ACAR and Fluorescence Spectroscopy. Phys. Chem. Chem. Phys. 2014, 16 (30), 15934. https://doi.org/10.1039/c4cp01386g.

(44) Piland, G. B.; Burdett, J. J.; Hung, T. Y.; Chen, P. H.; Lin, C. F.; Chiu, T. L.; Lee, J. H.; Bardeen, C. J. Dynamics of Molecular Excitons near a Semiconductor Surface Studied by Fluorescence Quenching of Polycrystalline Tetracene on Silicon. Chem. Phys. Lett. 2014, 601, 33-38. https://doi.org/10.1016/j.cplett.2014.03.075.

(45) Gieseking, B.; Schmeiler, T.; Müller, B.; Deibel, C.; Engels, B.; Dyakonov, V.; Pflaum, J. Effects of Characteristic Length Scales on the Exciton Dynamics in Rubrene Single Crystals. Phys. Rev. B - Condens. Matter Mater. Phys. 2014, 90 (20). https://doi.org/10.1103/PhysRevB.90.205305.

(46) Ryerson, J. L.; Schrauben, J. N.; Ferguson, A. J.; Sahoo, S. C.; Naumov, P.; Havlas, Z.; Michl, J.; Nozik, A. J.; Johnson, J. C. Two Thin Film Polymorphs of the Singlet Fission Compound 1,3Diphenylisobenzofuran. J. Phys. Chem. C 2014, 118 (23), 12121-12132. https://doi.org/10.1021/jp502122d.

(47) Johnson, J. C.; Nozik, A. J.; Michl, J. High Triplet Yield from Singlet Fission in a Thin Film of 1,3-Diphenylisobenzofuran. J. Am. Chem. Soc. 2010, 132 (46), 16302-16303. https://doi.org/10.1021/ja104123r.

(48) Akselrod, G. M.; Deotare, P. B.; Thompson, N. J.; Lee, J.; Tisdale, W. A.; Baldo, M. A.; Menon, V. M.; Bulovic, V. Visualization of Exciton Transport in Ordered and Disordered Molecular Solids. Nat. Commun. 2014, 5 (1). https://doi.org/10.1038/ncomms4646.

(49) Li, J.; Chen, Z.; Zhang, Q.; Xiong, Z.; Zhang, Y. Temperature-Dependent Singlet Exciton Fission Observed in Amorphous Rubrene Films. Org. Electron. 2015, 26, 213-217. https://doi.org/10.1016/j.orgel.2015.07.035.

(50) Li, J.; Chen, Z.; Lei, Y.; Xiong, Z.; Zhang, Y. Competition between Singlet Exciton Fission, Radiation, and Dissociation Measured in Rubrene-Doped Amorphous Films. Synth. Met. 2015, 207, 13-17. https://doi.org/10.1016/j.synthmet.2015.05.026.

(51) Thompson, N. J.; Hontz, E.; Chang, W.; Van Voorhis, T.; Baldo, M. Magnetic Feld Dependence 
of Singlet Fssion in Solutions of Diphenyl Tetracene. Philos. Trans. R. Soc. A Math. Phys. Eng. Sci. 2015, 373 (2044), 20140323. https://doi.org/10.1098/rsta.2014.0323.

(52) Lukman, S.; Musser, A. J.; Chen, K.; Athanasopoulos, S.; Yong, C. K.; Zeng, Z.; Ye, Q.; Chi, C.; Hodgkiss, J. M.; Wu, J.; et al. Tuneable Singlet Exciton Fission and Triplet-Triplet Annihilation in an Orthogonal Pentacene Dimer. Adv. Funct. Mater. 2015, 25 (34), 5452-5461. https://doi.org/10.1002/adfm.201501537.

(53) Wang, R.; Zhang, C.; Zhang, B.; Liu, Y.; Wang, X.; Xiao, M. Magnetic Dipolar Interaction between Correlated Triplets Created by Singlet Fission in Tetracene Crystals. Nat. Commun. 2015, 6 (1). https://doi.org/10.1038/ncomms9602.

(54) Varnavski, O.; Abeyasinghe, N.; Aragó, J.; Serrano-Pérez, J. J.; Ortí, E.; López Navarrete, J. T.; Takimiya, K.; Casanova, D.; Casado, J.; Goodson, T. High Yield Ultrafast Intramolecular Singlet Exciton Fission in a Quinoidal Bithiophene. J. Phys. Chem. Lett. 2015, 6 (8), 1375-1384. https://doi.org/10.1021/acs.jpclett.5b00198.

(55) Sanders, S. N.; Kumarasamy, E.; Pun, A. B.; Trinh, M. T.; Choi, B.; Xia, J.; Taffet, E. J.; Low, J. Z.; Miller, J. R.; Roy, X.; et al. Quantitative Intramolecular Singlet Fission in Bipentacenes. J. Am. Chem. Soc. 2015, 137 (28), 8965-8972. https://doi.org/10.1021/jacs.5b04986.

(56) Piland, G. B.; Bardeen, C. J. How Morphology Affects Singlet Fission in Crystalline Tetracene. J. Phys. Chem. Lett. 2015, 1841-1846. https://doi.org/10.1021/acs.jpclett.5b00569.

(57) Liu, H.; Nichols, V. M.; Shen, L.; Jahansouz, S.; Chen, Y.; Hanson, K. M.; Bardeen, C. J.; Li, X. Synthesis and Photophysical Properties of a "Face-to-Face" Stacked Tetracene Dimer. Phys.

Chem. Chem. Phys. 2015, 17 (9), 6523-6531. https://doi.org/10.1039/C4CP05444J.

(58) Schwerin, A. F.; Johnson, J. C.; Smith, M. B.; Sreearunothai, P.; Popović, D.; Černý, J.; Havias, Z.; Paci, I.; Akdag, A.; MacLeod, M. K.; et al. Toward Designed Singlet Fission: Electronic States and Photophysics of 1,3-Diphenylisobenzofuran. J. Phys. Chem. A 2010, 114 (3), 1457-1473. https://doi.org/10.1021/jp907401t.

(59) Ullah, M.; Yambem, S. D.; Moore, E. G.; Namdas, E. B.; Pandey, A. K. Singlet Fission and Triplet Exciton Dynamics in Rubrene/Fullerene Heterojunctions: Implications for Electroluminescence. Adv. Electron. Mater. 2015, 1 (12), 1500229. https://doi.org/10.1002/aelm.201500229.

(60) Nichols, V. M.; Broch, K.; Schreiber, F.; Bardeen, C. J. Excited-State Dynamics of Diindenoperylene in Liquid Solution and in Solid Films. J. Phys. Chem. C 2015, 119 (23), 1285612864. https://doi.org/10.1021/acs.jpcc.5b03353.

(61) Lukman, S.; Chen, K.; Hodgkiss, J. M.; Turban, D. H. P.; Hine, N. D. M.; Dong, S.; Wu, J.; Greenham, N. C.; Musser, A. J. Tuning the Role of Charge-Transfer States in Intramolecular Singlet Exciton Fission through Side-Group Engineering. Nat. Commun. 2016, 7 (1). https://doi.org/10.1038/ncomms13622.

(62) Margulies, E. A.; Miller, C. E.; Wu, Y.; Ma, L.; Schatz, G. C.; Young, R. M.; Wasielewski, M. R. Enabling Singlet Fission by Controlling Intramolecular Charge Transfer in $\pi$-Stacked Covalent Terrylenediimide Dimers. Nat. Chem. 2016, 8 (12), 1120-1125. https://doi.org/10.1038/nchem.2589.

(63) Schrauben, J. N.; Akdag, A.; Wen, J.; Havlas, Z.; Ryerson, J. L.; Smith, M. B.; Michl, J.; Johnson, J. C. Excitation Localization/Delocalization Isomerism in a Strongly Coupled Covalent Dimer of 1,3-Diphenylisobenzofuran. J. Phys. Chem. A 2016, 120 (20), 3473-3483. 
https://doi.org/10.1021/acs.jpca.6b00826.

(64) Tayebjee, M. J. Y.; Schwarz, K. N.; MacQueen, R. W.; Dvořák, M.; Lam, A. W. C.; Ghiggino, K. P.; McCamey, D. R.; Schmidt, T. W.; Conibeer, G. J. Morphological Evolution and Singlet Fission in Aqueous Suspensions of TIPS-Pentacene Nanoparticles. J. Phys. Chem. C 2016, 120 (1), 157-165. https://doi.org/10.1021/acs.jpcc.5b11353.

(65) Kato, D.; Sakai, H.; Tkachenko, N. V.; Hasobe, T. High-Yield Excited Triplet States in Pentacene Self-Assembled Monolayers on Gold Nanoparticles through Singlet Exciton Fission. Angew. Chemie - Int. Ed. 2016, 55 (17), 5230-5234. https://doi.org/10.1002/anie.201601421.

(66) Lin, Y. H. L.; Fusella, M. A.; Kozlov, O. V.; Lin, X.; Kahn, A.; Pshenichnikov, M. S.; Rand, B. P. Morphological Tuning of the Energetics in Singlet Fission Organic Solar Cells. Adv. Funct. Mater. 2016, 26 (35), 6489-6494. https://doi.org/10.1002/adfm.201601125.

(67) Fuemmeler, E. G.; Sanders, S. N.; Pun, A. B.; Kumarasamy, E.; Zeng, T.; Miyata, K.; Steigerwald, M. L.; Zhu, X.-Y.; Sfeir, M. Y.; Campos, L. M.; et al. A Direct Mechanism of Ultrafast Intramolecular Singlet Fission in Pentacene Dimers. ACS Cent. Sci. 2016, 2 (5), 316-324. https://doi.org/10.1021/acscentsci.6b00063.

(68) Korovina, N. V.; Das, S.; Nett, Z.; Feng, X.; Joy, J.; Haiges, R.; Krylov, A. I.; Bradforth, S. E.; Thompson, M. E. Singlet Fission in a Covalently Linked Cofacial Alkynyltetracene Dimer. J. Am. Chem. Soc. 2016, 138 (2), 617-627. https://doi.org/10.1021/jacs.5b10550.

(69) Burdett, J. J.; Müller, A. M.; Gosztola, D.; Bardeen, C. J. Excited State Dynamics in Solid and Monomeric Tetracene: The Roles of Superradiance and Exciton Fission. J. Chem. Phys. 2010, 133 (14), 144506. https://doi.org/10.1063/1.3495764.

(70) Sanders, S. N.; Kumarasamy, E.; Pun, A. B.; Appavoo, K.; Steigerwald, M. L.; Campos, L. M.; Sfeir, M. Y. Exciton Correlations in Intramolecular Singlet Fission. J. Am. Chem. Soc. 2016, 138 (23), 7289-7297. https://doi.org/10.1021/jacs.6b00657.

(71) Le, A. K.; Bender, J. A.; Roberts, S. T. Slow Singlet Fission Observed in a Polycrystalline Perylenediimide Thin Film. J. Phys. Chem. Lett. 2016, 7 (23), 4922-4928.

https://doi.org/10.1021/acs.jpclett.6b02320.

(72) Cook, J. D.; Carey, T. J.; Damrauer, N. H. Solution-Phase Singlet Fission in a Structurally WellDefined Norbornyl-Bridged Tetracene Dimer. J. Phys. Chem. A 2016, 120 (26), 4473-4481. https://doi.org/10.1021/acs.jpca.6b04367.

(73) Mauck, C. M.; Hartnett, P. E.; Margulies, E. A.; Ma, L.; Miller, C. E.; Schatz, G. C.; Marks, T. J.; Wasielewski, M. R. Singlet Fission via an Excimer-Like Intermediate in 3,6-Bis(Thiophen-2yl)Diketopyrrolopyrrole Derivatives. J. Am. Chem. Soc. 2016, 138 (36), 11749-11761. https://doi.org/10.1021/jacs.6b05627.

(74) Zhang, Y.-D.; Wu, Y.; Xu, Y.; Wang, Q.; Liu, K.; Chen, J.-W.; Cao, J.-J.; Zhang, C.; Fu, H.; Zhang, H.-L. Excessive Exoergicity Reduces Singlet Exciton Fission Efficiency of Heteroacenes in Solutions. J. Am. Chem. Soc. 2016, 138 (21), 6739-6745. https://doi.org/10.1021/jacs.6b03829.

(75) Yago, T.; Ishikawa, K.; Katoh, R.; Wakasa, M. Magnetic Field Effects on Triplet Pair Generated by Singlet Fission in an Organic Crystal: Application of Radical Pair Model to Triplet Pair. $J$. Phys. Chem. C 2016, 120 (49), 27858-27870. https://doi.org/10.1021/acs.jpcc.6b09570.

(76) Sun, T.; Shen, L.; Liu, H.; Sun, X.; Li, X. Synthesis and Photophysical Properties of a Single Bond Linked Tetracene Dimer. J. Mol. Struct. 2016, 1116, 200-206. https://doi.org/10.1016/j.molstruc.2016.03.048. 
(77) Sakuma, T.; Sakai, H.; Araki, Y.; Mori, T.; Wada, T.; Tkachenko, N. V.; Hasobe, T. Long-Lived Triplet Excited States of Bent-Shaped Pentacene Dimers by Intramolecular Singlet Fission. $J$. Phys. Chem. A 2016, 120 (11), 1867-1875. https://doi.org/10.1021/acs.jpca.6b00988.

(78) Le, A. K.; Bender, J. A.; Arias, D. H.; Cotton, D. E.; Johnson, J. C.; Roberts, S. T. Singlet Fission Involves an Interplay between Energetic Driving Force and Electronic Coupling in Perylenediimide Films. J. Am. Chem. Soc. 2018, 140 (2), 814-826. https://doi.org/10.1021/jacs.7b11888.

(79) Tian, X.; Zhou, L.; Chen, X.; Meng, Y.; Xiong, Z.; Zhou, X.; Zhang, Y. Nanoscale Exponential Distance Dependence and Electron-Transfer Model for Intermolecular Singlet Exciton Fission Observed in Rubrene-Doped Organic Films. Org. Electron. 2017, 50, 429-434. https://doi.org/10.1016/j.orgel.2017.08.009.

(80) Najafov, H.; Lee, B.; Zhou, Q.; Feldman, L. C.; Podzorov, V. Observation of Long-Range Exciton Diffusion in Highly Ordered Organic Semiconductors. Nat. Mater. 2010, 9 (11), 938-943. https://doi.org/10.1038/nmat2872.

(81) Katoh, R.; Hashimoto, M.; Takahashi, A.; Sonoda, Y.; Yago, T.; Wakasa, M. Singlet Fission in Fluorinated Diphenylhexatrienes. J. Phys. Chem. C 2017, 121 (46), 25666-25671. https://doi.org/10.1021/acs.jpcc.7b06905.

(82) Piland, G. B.; Bardeen, C. J. Photoluminescence Dynamics in Singlet Fission Chromophore Liquid Melts. Chem. Phys. Lett. 2017, 669, 99-103. https://doi.org/10.1016/j.cplett.2016.12.021.

(83) Izadnia, S.; Schönleber, D. W.; Eisfeld, A.; Ruf, A.; LaForge, A. C.; Stienkemeier, F. Singlet Fission in Weakly Interacting Acene Molecules. J. Phys. Chem. Lett. 2017, 8 (9), 2068-2073. https://doi.org/10.1021/acs.jpclett.7b00319.

(84) Dean, J. C.; Zhang, R.; Hallani, R. K.; Pensack, R. D.; Sanders, S. N.; Oblinsky, D. G.; Parkin, S. R.; Campos, L. M.; Anthony, J. E.; Scholes, G. D. Photophysical Characterization and TimeResolved Spectroscopy of a Anthradithiophene Dimer: Exploring the Role of Conformation in Singlet Fission. Phys. Chem. Chem. Phys. 2017, 19 (34), 23162-23175. https://doi.org/10.1039/c7cp03774k.

(85) Thampi, A.; Stern, H. L.; Cheminal, A.; Tayebjee, M. J. Y.; Petty, A. J.; Anthony, J. E.; Rao, A. Elucidation of Excitation Energy Dependent Correlated Triplet Pair Formation Pathways in an Endothermic Singlet Fission System. J. Am. Chem. Soc. 2018, 140 (13), 4613-4622. https://doi.org/10.1021/jacs.7b06274.

(86) Stern, H. L.; Cheminal, A.; Yost, S. R.; Broch, K.; Bayliss, S. L.; Chen, K.; Tabachnyk, M.; Thorley, K.; Greenham, N.; Hodgkiss, J. M.; et al. Vibronically Coherent Ultrafast Triplet-Pair Formation and Subsequent Thermally Activated Dissociation Control Efficient Endothermic Singlet Fission. Nat. Chem. 2017, 9 (12), 1205-1212. https://doi.org/10.1038/nchem.2856.

(87) Liu, H.; Wang, R.; Shen, L.; Xu, Y.; Xiao, M.; Zhang, C.; Li, X. A Covalently Linked Tetracene Trimer: Synthesis and Singlet Exciton Fission Property. Org. Lett. 2017, 19 (3), 580-583. https://doi.org/10.1021/acs.orglett.6b03739.

(88) Lukman, S.; Richter, J. M.; Yang, L.; Hu, P.; Wu, J.; Greenham, N. C.; Musser, A. J. Efficient Singlet Fission and Triplet-Pair Emission in a Family of Zethrene Diradicaloids. J. Am. Chem. Soc. 2017, 139 (50), 18376-18385. https://doi.org/10.1021/jacs.7b10762.

(89) Pun, A. B.; Sanders, S. N.; Kumarasamy, E.; Sfeir, M. Y.; Congreve, D. N.; Campos, L. M. Triplet Harvesting from Intramolecular Singlet Fission in Polytetracene. Adv. Mater. 2017, 29 (41), 
1701416. https://doi.org/10.1002/adma.201701416.

(90) Yong, C. K.; Musser, A. J.; Bayliss, S. L.; Lukman, S.; Tamura, H.; Bubnova, O.; Hallani, R. K.; Meneau, A.; Resel, R.; Maruyama, M.; et al. The Entangled Triplet Pair State in Acene and Heteroacene Materials. Nat. Commun. 2017, 8 (1). https://doi.org/10.1038/ncomms15953.

(91) Burdett, J. J.; Gosztola, D.; Bardeen, C. J. The Dependence of Singlet Exciton Relaxation on Excitation Density and Temperature in Polycrystalline Tetracene Thin Films: Kinetic Evidence for a Dark Intermediate State and Implications for Singlet Fission. J. Chem. Phys. 2011, 135 (21), 214508. https://doi.org/10.1063/1.3664630.

(92) Dron, P. I.; Michl, J.; Johnson, J. C. Singlet Fission and Excimer Formation in Disordered Solids of Alkyl-Substituted 1,3-Diphenylisobenzofurans. J. Phys. Chem. A 2017, 121 (45), 8596-8603. https://doi.org/10.1021/acs.jpca.7b07362.

(93) Liu, H.; Wang, X.; Pan, L.; Shen, L.; Wang, X.; Chen, Q.; Li, X. Synthesis and Photophysical Properties of a Bistetracene Compound with Slipped Stacked Structure. J. Photochem. Photobiol. A Chem. 2017, 340, 21-28. https://doi.org/10.1016/j.jphotochem.2017.03.006.

(94) Aulin, Y. V.; Felter, K. M.; Günbas, D. D.; Dubey, R. K.; Jager, W. F.; Grozema, F. C. Morphology-Independent Efficient Singlet Exciton Fission in Perylene Diimide Thin Films. Chempluschem 2018, 83 (4), 230-238. https://doi.org/10.1002/cplu.201700449.

(95) Tilley, A. J.; Pensack, R. D.; Kynaston, E. L.; Scholes, G. D.; Seferos, D. S. Singlet Fission in Core-Shell Micelles of End-Functionalized Polymers. Chem. Mater. 2018, 30 (13), 4409-4421. https://doi.org/10.1021/acs.chemmater.8b01814.

(96) Dover, C. B.; Gallaher, J. K.; Frazer, L.; Tapping, P. C.; Petty, A. J.; Crossley, M. J.; Anthony, J. E.; Kee, T. W.; Schmidt, T. W. Endothermic Singlet Fission Is Hindered by Excimer Formation. Nat. Chem. 2018, 10 (3), 305-310. https://doi.org/10.1038/nchem.2926.

(97) Manna, B.; Nandi, A.; Ghosh, R. Ultrafast Singlet Exciton Fission Dynamics in 9,10Bis(Phenylethynyl)Anthracene Nanoaggregates and Thin Films. J. Phys. Chem. C 2018, 122 (36), 21047-21055. https://doi.org/10.1021/acs.jpcc.8b05260.

(98) Masoomi-Godarzi, S.; Liu, M.; Tachibana, Y.; Goerigk, L.; Ghiggino, K. P.; Smith, T. A.; Jones, D. J. Solution-Processable, Solid State Donor-Acceptor Materials for Singlet Fission. Adv. Energy Mater. 2018, 8 (30), 1801720. https://doi.org/10.1002/aenm.201801720.

(99) Pun, J. K. H.; Gallaher, J. K.; Frazer, L.; Prasad, S. K. K.; Dover, C. B.; MacQueen, R. W.; Schmidt, T. W. TIPS-Anthracene: A Singlet Fission or Triplet Fusion Material? J. Photonics Energy 2018, 8 (02), 1. https://doi.org/10.1117/1.jpe.8.022006.

(100) Lavarda, G.; Zirzlmeier, J.; Gruber, M.; Rami, P. R.; Tykwinski, R. R.; Torres, T.; Guldi, D. M. Tuning Intramolecular Förster Resonance Energy Transfer and Activating Intramolecular Singlet Fission. Angew. Chemie - Int. Ed. 2018, 57 (50), 16291-16295. https://doi.org/10.1002/anie.201808652.

(101) Yamakado, T.; Takahashi, S.; Watanabe, K.; Matsumoto, Y.; Osuka, A.; Saito, S. Conformational Planarization versus Singlet Fission: Distinct Excited-State Dynamics of Cyclooctatetraene-Fused Acene Dimers. Angew. Chemie Int. Ed. 2018, 57 (19), 5438-5443. https://doi.org/10.1002/anie.201802185.

(102) Ma, L.; Zhang, K.; Kloc, C.; Sun, H.; Michel-Beyerle, M. E.; Gurzadyan, G. G. Singlet Fission in Rubrene Single Crystal: Direct Observation by Femtosecond Pump-Probe Spectroscopy. Phys. Chem. Chem. Phys. 2012, 14 (23), 8307. https://doi.org/10.1039/c2cp40449d. 
(103) Pensack, R. D.; Tilley, A. J.; Grieco, C.; Purdum, G. E.; Ostroumov, E. E.; Granger, D. B.; Oblinsky, D. G.; Dean, J. C.; Doucette, G. S.; Asbury, J. B.; et al. Striking the Right Balance of Intermolecular Coupling for High-Efficiency Singlet Fission. Chem. Sci. 2018, 9 (29), 6240-6259. https://doi.org/10.1039/c8sc00293b.

(104) Grieco, C.; Kennehan, E. R.; Kim, H.; Pensack, R. D.; Brigeman, A. N.; Rimshaw, A.; Payne, M. M.; Anthony, J. E.; Giebink, N. C.; Scholes, G. D.; et al. Direct Observation of Correlated Triplet Pair Dynamics during Singlet Fission Using Ultrafast Mid-IR Spectroscopy. J. Phys. Chem. C 2018, 122 (4), 2012-2022. https://doi.org/10.1021/acs.jpcc.7b11228.

(105) Banerjee, T.; Hill, S. P.; Hermosilla-Palacios, M. A.; Piercy, B. D.; Haney, J.; Casale, B.; Deprince, A. E.; Losego, M. D.; Kleiman, V. D.; Hanson, K. Diphenylisobenzofuran Bound to Nanocrystalline Metal Oxides: Excimer Formation, Singlet Fission, Electron Injection, and Low Energy Sensitization. J. Phys. Chem. C 2018, 122 (50), 28478-28490. https://doi.org/10.1021/acs.jpcc.8b08599.

(106) Bae, Y. J.; Kang, G.; Malliakas, C. D.; Nelson, J. N.; Zhou, J.; Young, R. M.; Wu, Y. L.; Van Duyne, R. P.; Schatz, G. C.; Wasielewski, M. R. Singlet Fission in 9,10Bis(Phenylethynyl)Anthracene Thin Films. J. Am. Chem. Soc. 2018, 140 (45), 15140-15144. https://doi.org/10.1021/jacs.8b07498.

(107) Nagashima, H.; Kawaoka, S.; Akimoto, S.; Tachikawa, T.; Matsui, Y.; Ikeda, H.; Kobori, Y. Singlet-Fission-Born Quintet State: Sublevel Selections and Trapping by Multiexciton Thermodynamics. J. Phys. Chem. Lett. 2018, 9 (19), 5855-5861. https://doi.org/10.1021/acs.jpclett.8b02396.

(108) Hu, J.; Xu, K.; Shen, L.; Wu, Q.; He, G.; Wang, J. Y.; Pei, J.; Xia, J.; Sfeir, M. Y. New Insights into the Design of Conjugated Polymers for Intramolecular Singlet Fission. Nat. Commun. 2018, 9 (1). https://doi.org/10.1038/s41467-018-05389-w.

(109) Chen, M.; Bae, Y. J.; Mauck, C. M.; Mandal, A.; Young, R. M.; Wasielewski, M. R. Singlet Fission in Covalent Terrylenediimide Dimers: Probing the Nature of the Multiexciton State Using Femtosecond Mid-Infrared Spectroscopy. J. Am. Chem. Soc. 2018, 140 (29), 9184-9192. https://doi.org/10.1021/jacs.8b04830.

(110) Mauck, C. M.; Bae, Y. J.; Chen, M.; Powers-Riggs, N.; Wu, Y.-L.; Wasielewski, M. R. ChargeTransfer Character in a Covalent Diketopyrrolopyrrole Dimer: Implications for Singlet Fission. ChemPhotoChem 2018, 2 (3), 223-233. https://doi.org/10.1002/cptc.201700135.

(111) Liu, H.; Wang, Z.; Wang, X.; Shen, L.; Zhang, C.; Xiao, M.; Li, X. Singlet Exciton Fission in a Linear Tetracene Tetramer. J. Mater. Chem. C 2018, 6 (13), 3245-3253. https://doi.org/10.1039/c7tc05783k.

(112) Montero, R.; Martínez-Martínez, V.; Longarte, A.; Epelde-Elezcano, N.; Palao, E.; Lamas, I.; Manzano, H.; Agarrabeitia, A. R.; López Arbeloa, I.; Ortiz, M. J.; et al. Singlet Fission Mediated Photophysics of BODIPY Dimers. J. Phys. Chem. Lett. 2018, 9 (3), 641-646. https://doi.org/10.1021/acs.jpclett.7b03074.

(113) Roberts, S. T.; McAnally, R. E.; Mastron, J. N.; Webber, D. H.; Whited, M. T.; Brutchey, R. L.; Thompson, M. E.; Bradforth, S. E. Efficient Singlet Fission Discovered in a Disordered Acene Film. J. Am. Chem. Soc. 2012, 134 (14), 6388-6400. https://doi.org/10.1021/ja300504t.

(114) Wolf, E. A.; Finton, D. M.; Zoutenbier, V.; Biaggio, I. Quantum Beats of a Multiexciton State in Rubrene Single Crystals. Appl. Phys. Lett. 2018, 112 (8), 083301. https://doi.org/10.1063/1.5020652. 
(115) Conrad-Burton, F. S.; Liu, T.; Geyer, F.; Costantini, R.; Schlaus, A. P.; Spencer, M. S.; Wang, J.; Sánchez, R. H.; Zhang, B.; Xu, Q.; et al. Controlling Singlet Fission by Molecular Contortion. J. Am. Chem. Soc. 2019, 141 (33), 13143-13147. https://doi.org/10.1021/jacs.9b05357.

(116) Sher, P. H.; Chen, C. H.; Chiu, T. L.; Lin, C. F.; Wang, J. K.; Lee, J. H. Distinct Routes of Singlet Fission and Triplet Fusion: A Fluorescence Kinetic Study of Rubrene. J. Phys. Chem. C 2019, 123 (6), 3279-3284. https://doi.org/10.1021/acs.jpcc.8b08677.

(117) Yablon, L. M.; Sanders, S. N.; Li, H.; Parenti, K. R.; Kumarasamy, E.; Fallon, K. J.; Hore, M. J. A.; Cacciuto, A.; Sfeir, M. Y.; Campos, L. M. Persistent Multiexcitons from Polymers with Pendent Pentacenes. J. Am. Chem. Soc. 2019, 141 (24), 9564-9569. https://doi.org/10.1021/jacs.9b02241.

(118) Shen, C.; Yan, S.; Chen, X.; Niu, L.; Zhang, Y. Magnetic Field Effect on Singlet Exciton Fission: A Sensitive Probe of Molecular Level Morphology in Organic Films. Org. Electron. 2019, 67, 194-199. https://doi.org/10.1016/j.orgel.2019.01.021.

(119) Wollscheid, N.; Pérez Lustres, J. L.; Kefer, O.; Hahn, S.; Brosius, V.; Bunz, U. H. F.; Motzkus, M.; Buckup, T. Oxygen-Catalysed Sequential Singlet Fission. Nat. Commun. 2019, 10 (1). https://doi.org/10.1038/s41467-019-13202-5.

(120) Ni, W.; Gurzadyan, G. G.; Zhao, J.; Che, Y.; Li, X.; Sun, L. Singlet Fission from Upper Excited Electronic States of Cofacial Perylene Dimer. J. Phys. Chem. Lett. 2019, 10 (10), 2428-2433. https://doi.org/10.1021/acs.jpclett.9b00717.

(121) Fallon, K. J.; Budden, P.; Salvadori, E.; Ganose, A. M.; Savory, C. N.; Eyre, L.; Dowland, S.; Ai, Q.; Goodlett, S.; Risko, C.; et al. Exploiting Excited-State Aromaticity to Design Highly Stable Singlet Fission Materials. J. Am. Chem. Soc. 2019, 141 (35), 13867-13876. https://doi.org/10.1021/jacs.9b06346.

(122) Allardice, J. R.; Thampi, A.; Dowland, S.; Xiao, J.; Gray, V.; Zhang, Z.; Budden, P.; Petty, A. J.; Davis, N. J. L. K.; Greenham, N. C.; et al. Engineering Molecular Ligand Shells on Quantum Dots for Quantitative Harvesting of Triplet Excitons Generated by Singlet Fission. J. Am. Chem. Soc. 2019, 141 (32), 12907-12915. https://doi.org/10.1021/jacs.9b06584.

(123) Einzinger, M.; Wu, T.; Kompalla, J. F.; Smith, H. L.; Perkinson, C. F.; Nienhaus, L.; Wieghold, S.; Congreve, D. N.; Kahn, A.; Bawendi, M. G.; et al. Sensitization of Silicon by Singlet Exciton Fission in Tetracene. Nature 2019, 571 (7763), 90-94. https://doi.org/10.1038/s41586-019-1339-4.

(124) Bakulin, A. A.; Morgan, S. E.; Kehoe, T. B.; Wilson, M. W. B.; Chin, A. W.; Zigmantas, D.; Egorova, D.; Rao, A. Real-Time Observation of Multiexcitonic States in Ultrafast Singlet Fission Using Coherent 2D Electronic Spectroscopy. Nat. Chem. 2016, 8 (1), 16-23. https://doi.org/10.1038/nchem.2371.

(125) Breen, I.; Tempelaar, R.; Bizimana, L. A.; Kloss, B.; Reichman, D. R.; Turner, D. B. Triplet Separation Drives Singlet Fission after Femtosecond Correlated Triplet Pair Production in Rubrene. J. Am. Chem. Soc. 2017, 139 (34), 11745-11751. https://doi.org/10.1021/jacs.7b02621.

(126) Mandal, A.; Chen, M.; Foszcz, E. D.; Schultz, J. D.; Kearns, N. M.; Young, R. M.; Zanni, M. T.; Wasielewski, M. R. Two-Dimensional Electronic Spectroscopy Reveals Excitation EnergyDependent State Mixing during Singlet Fission in a Terrylenediimide Dimer. J. Am. Chem. Soc. 2018, 140 (51), 17907-17914. https://doi.org/10.1021/jacs.8b08627.

(127) Chan, W. L.; Ligges, M.; Jailaubekov, A.; Kaake, L.; Miaja-Avila, L.; Zhu, X. Y. Observing the Multiexciton State in Singlet Fission and Ensuing Ultrafast Multielectron Transfer. Science. 2011, 
334 (6062), 1541-1545. https://doi.org/10.1126/science.1213986.

(128) Chan, W.-L.; Ligges, M.; Zhu, X.-Y. The Energy Barrier in Singlet Fission Can Be Overcome through Coherent Coupling and Entropic Gain. Nat. Chem. 2012, 4 (10), 840-845.

https://doi.org/10.1038/nchem.1436.

(129) Chan, W. L.; Tritsch, J. R.; Zhu, X. Y. Harvesting Singlet Fission for Solar Energy Conversion: One- Versus Two-Electron Transfer from the Quantum Mechanical Superposition. J. Am. Chem. Soc. 2012, 134 (44), 18295-18302. https://doi.org/10.1021/ja306271y.

(130) Tritsch, J. R.; Chan, W. L.; Wu, X.; Monahan, N. R.; Zhu, X. Y. Harvesting Singlet Fission for Solar Energy Conversion via Triplet Energy Transfer. Nat. Commun. 2013, 4 (1). https://doi.org/10.1038/ncomms3679.

(131) Monahan, N. R.; Sun, D.; Tamura, H.; Williams, K. W.; Xu, B.; Zhong, Y.; Kumar, B.; Nuckolls, C.; Harutyunyan, A. R.; Chen, G.; et al. Dynamics of the Triplet-Pair State Reveals the Likely Coexistence of Coherent and Incoherent Singlet Fission in Crystalline Hexacene. Nat. Chem. 2017, 9 (4), 341-346. https://doi.org/10.1038/nchem.2665.

(132) Stein, A.; Maaß, F.; Tegeder, P. Triisopropylsilylethynyl-Pentacene on Au(111): Adsorption Properties, Electronic Structure, and Singlet Fission Dynamics. J. Phys. Chem. C 2017, 121 (33), 18075-18083. https://doi.org/10.1021/acs.jpcc.7b06340.

(133) Nagashima, H.; Kawaoka, S.; Matsui, Y.; Tachikawa, T.; Ikeda, H.; Kobori, Y. Time-Resolved EPR Study on Singlet-Fission Induced Quintet Generation and Subsequent Triplet Dissociation in TIPS-Phenyl-Tetracene Aggregates. J. Photopolym. Sci. Technol. 2018, 31 (2), 163-167. https://doi.org/10.2494/photopolymer.31.163.

(134) Lubert-Perquel, D.; Salvadori, E.; Dyson, M.; Stavrinou, P. N.; Montis, R.; Nagashima, H.; Kobori, Y.; Heutz, S.; Kay, C. W. M. Identifying Triplet Pathways in Dilute Pentacene Films. Nat. Commun. 2018, 9 (1). https://doi.org/10.1038/s41467-018-06330-x.

(135) Bayliss, S. L.; Kraffert, F.; Wang, R.; Zhang, C.; Bittl, R.; Behrends, J. Tuning Spin Dynamics in Crystalline Tetracene. J. Phys. Chem. Lett. 2019, 10 (8), 1908-1913. https://doi.org/10.1021/acs.jpclett.9b00356.

(136) Jue Bae, Y.; Krzyaniak, M. D.; Majewski, M. B.; Desroches, M.; Morin, J. F.; Wu, Y. L.; Wasielewski, M. R. Competition between Singlet Fission and Spin-Orbit-Induced Intersystem Crossing in Anthanthrene and Anthanthrone Derivatives. Chempluschem 2019, 84 (9), 1432-1438. https://doi.org/10.1002/cplu.201900410.

(137) Pun, A. B.; Asadpoordarvish, A.; Kumarasamy, E.; Tayebjee, M. J. Y.; Niesner, D.; McCamey, D. R.; Sanders, S. N.; Campos, L. M.; Sfeir, M. Y. Ultra-Fast Intramolecular Singlet Fission to Persistent Multiexcitons by Molecular Design. Nat. Chem. 2019, 11 (9), 821-828. https://doi.org/10.1038/s41557-019-0297-7.

(138) Saegusa, T.; Sakai, H.; Nagashima, H.; Kobori, Y.; Tkachenko, N. V.; Hasobe, T. Controlled Orientations of Neighboring Tetracene Units by Mixed Self-Assembled Monolayers on Gold Nanoclusters for High-Yield and Long-Lived Triplet Excited States through Singlet Fission. $J$. Am. Chem. Soc. 2019, 141 (37), 14720-14727. https://doi.org/10.1021/jacs.9b06567.

(139) Matsuda, S.; Oyama, S.; Kobori, Y. Electron Spin Polarization Generated by Transport of Singlet and Quintet Multiexcitons to Spin-Correlated Triplet Pairs during Singlet Fissions. Chem. Sci. 2020, 11 (11), 2934-2942. https://doi.org/10.1039/c9sc04949e.

(140) Basel, B. S.; Zirzlmeier, J.; Hetzer, C.; Phelan, B. T.; Krzyaniak, M. D.; Reddy, S. R.; Coto, P. B.; 
Horwitz, N. E.; Young, R. M.; White, F. J.; et al. Unified Model for Singlet Fission within a NonConjugated Covalent Pentacene Dimer. Nat. Commun. 2017, 8 (1).

https://doi.org/10.1038/ncomms15171.

(141) Tayebjee, M. J. Y.; Sanders, S. N.; Kumarasamy, E.; Campos, L. M.; Sfeir, M. Y.; McCamey, D. R. Quintet Multiexciton Dynamics in Singlet Fission. Nat. Phys. 2017, 13 (2), 182-188. https://doi.org/10.1038/nphys3909.

(142) Weiss, L. R.; Bayliss, S. L.; Kraffert, F.; Thorley, K. J.; Anthony, J. E.; Bittl, R.; Friend, R. H.; Rao, A.; Greenham, N. C.; Behrends, J. Strongly Exchange-Coupled Triplet Pairs in an Organic Semiconductor. Nat. Phys. 2017, 13 (2), 176-181. https://doi.org/10.1038/nphys3908.

(143) Basel, B. S.; Zirzlmeier, J.; Hetzer, C.; Reddy, S. R.; Phelan, B. T.; Krzyaniak, M. D.; Volland, M. K.; Coto, P. B.; Young, R. M.; Clark, T.; et al. Evidence for Charge-Transfer Mediation in the Primary Events of Singlet Fission in a Weakly Coupled Pentacene Dimer. Chem 2018, 4 (5), 1092-1111. https://doi.org/10.1016/j.chempr.2018.04.006.

(144) Grieco, C.; Kennehan, E. R.; Rimshaw, A.; Payne, M. M.; Anthony, J. E.; Asbury, J. B. Harnessing Molecular Vibrations to Probe Triplet Dynamics during Singlet Fission. J. Phys. Chem. Lett. 2017, 8 (23), 5700-5706. https://doi.org/10.1021/acs.jpclett.7b02434.

(145) Deng, G. H.; Wei, Q.; Han, J.; Qian, Y.; Luo, J.; Harutyunyan, A. R.; Chen, G.; Bian, H.; Chen, H.; Rao, Y. Vibronic Fingerprint of Singlet Fission in Hexacene. J. Chem. Phys. 2019, 151 (5), 054703. https://doi.org/10.1063/1.5110263.

(146) Grieco, C.; Doucette, G. S.; Munson, K. T.; Swartzfager, J. R.; Munro, J. M.; Anthony, J. E.; Dabo, I.; Asbury, J. B. Vibrational Probe of the Origin of Singlet Exciton Fission in TIPSPentacene Solutions. J. Chem. Phys. 2019, 151 (15), 154701. https://doi.org/10.1063/1.5116586.

(147) Grumstrup, E. M.; Johnson, J. C.; Damrauer, N. H. Enhanced Triplet Formation in Polycrystalline Tetracene Films by Femtosecond Optical-Pulse Shaping. Phys. Rev. Lett. 2010, 105 (25). https://doi.org/10.1103/PhysRevLett.105.257403.

(148) Musser, A. J.; Liebel, M.; Schnedermann, C.; Wende, T.; Kehoe, T. B.; Rao, A.; Kukura, P. Evidence for Conical Intersection Dynamics Mediating Ultrafast Singlet Exciton Fission. Nat. Phys. 2015, 11 (4), 352-357. https://doi.org/10.1038/nphys3241.

(149) Schnedermann, C.; Alvertis, A. M.; Wende, T.; Lukman, S.; Feng, J.; Schröder, F. A. Y. N.; Turban, D. H. P.; Wu, J.; Hine, N. D. M.; Greenham, N. C.; et al. A Molecular Movie of Ultrafast Singlet Fission. Nat. Commun. 2019, 10 (1), 1-11. https://doi.org/10.1038/s41467-019-12220-7.

(150) Wang, C.; Tauber, M. J. High-Yield Singlet Fission in a Zeaxanthin Aggregate Observed by Picosecond Resonance Raman Spectroscopy. J. Am. Chem. Soc. 2010, 132 (40), 13988-13991. https://doi.org/10.1021/ja102851m.

(151) Wang, C.; Schlamadinger, D. E.; Desai, V.; Tauber, M. J. Triplet Excitons of Carotenoids Formed by Singlet Fission in a Membrane. ChemPhysChem 2011, 12 (16), 2891-2894. https://doi.org/10.1002/cphc.201100571.

(152) Hart, S. M.; Silva, W. R.; Frontiera, R. R. Femtosecond Stimulated Raman Evidence for ChargeTransfer Character in Pentacene Singlet Fission. Chem. Sci. 2018, 9 (5), 1242-1250. https://doi.org/10.1039/c7sc03496b.

(153) Bera, K.; Douglas, C. J.; Frontiera, R. R. Femtosecond Raman Microscopy Reveals Structural Dynamics Leading to Triplet Separation in Rubrene Singlet Fission. J. Phys. Chem. Lett. 2017, 8 (23), 5929-5934. https://doi.org/10.1021/acs.jpclett.7b02769. 
(154) Jana, S.; Yapamanu, A. L.; Umapathy, S. Unraveling Structural Dynamics in Isoenergetic Excited $\mathrm{S}_{1}$ and Multi-Excitonic ${ }^{1}$ (TT) States of 9,10-Bis(Phenylethynyl)Anthracene (BPEA) in Solution: Via Ultrafast Raman Loss Spectroscopy. Phys. Chem. Chem. Phys. 2019, 21 (26), 14341-14349. https://doi.org/10.1039/c8cp06658b.

(155) Bera, K.; Douglas, C. J.; Frontiera, R. R. Femtosecond Stimulated Raman Spectroscopy - Guided Screening Leads to Efficient Singlet Fission in Rubrene Derivatives. Submitt. to Chem. Sci. 2020.

(156) Wang, X.; Wang, R.; Shen, L.; Tang, Z.; Wen, C.; Dong, B.; Liu, H.; Zhang, C.; Li, X. Intramolecular Singlet Fission in a Face-to-Face Stacked Tetracene Trimer. Phys. Chem. Chem. Phys. 2018, 20 (9), 6330-6336. https://doi.org/10.1039/c7cp07841b.

(157) Alvertis, A. M.; Lukman, S.; Hele, T. J. H.; Fuemmeler, E. G.; Feng, J.; Wu, J.; Greenham, N. C.; Chin, A. W.; Musser, A. J. Switching between Coherent and Incoherent Singlet Fission via Solvent-Induced Symmetry Breaking. J. Am. Chem. Soc. 2019, 141 (44), 17558-17570. https://doi.org/10.1021/jacs.9b05561.

(158) Thompson, N. J.; Wilson, M. W. B.; Congreve, D. N.; Brown, P. R.; Scherer, J. M.; Bischof, T. S.; Wu, M.; Geva, N.; Welborn, M.; Van Voorhis, T.; et al. Energy Harvesting of Non-Emissive Triplet Excitons in Tetracene by Emissive PbS Nanocrystals. Nat. Mat. 2018, 13 (11), 1039-1043. https://doi.org/10.1038/nmat4097.

(159) Lee, S.; Hwang, D.; Jung, S. Il; Kim, D. Electron Transfer from Triplet State of TIPS-Pentacene Generated by Singlet Fission Processes to $\mathrm{CH}_{3} \mathrm{NH}_{3} \mathrm{PbI}_{3}$ Perovskite. J. Phys. Chem. Lett. 2017, 8 (4), 884-888. https://doi.org/10.1021/acs.jpclett.7b00072.

(160) Davis, N. J. L. K.; Allardice, J. R.; Xiao, J.; Petty, A. J.; Greenham, N. C.; Anthony, J. E.; Rao, A. Singlet Fission and Triplet Transfer to PbS Quantum Dots in TIPS-Tetracene Carboxylic Acid Ligands. J. Phys. Chem. Lett. 2018, 9 (6), 1454-1460. https://doi.org/10.1021/acs.jpclett.8b00099.

(161) Nagata, R.; Nakanotani, H.; Potscavage, W. J.; Adachi, C. Exploiting Singlet Fission in Organic Light-Emitting Diodes. Adv. Mater. 2018, 30 (33), 1801484. https://doi.org/10.1002/adma.201801484.

(162) Tabernig, S. W.; Daiber, B.; Wang, T.; Ehrler, B. Enhancing Silicon Solar Cells with Singlet Fission: The Case for Förster Resonant Energy Transfer Using a Quantum Dot Intermediate. J. Photonics Energy 2018, 8 (02), 1. https://doi.org/10.1117/1.jpe.8.022008.

(163) Niu, M. S.; Yang, X. Y.; Qin, C. C.; Bi, P. Q.; Lyu, C. K.; Feng, L.; Qin, W.; Gao, K.; Hao, X. T. Competition between Singlet Fission and Singlet Exciton Dissociation at the Interface in TIPSPentacene:IT-4F Blend. Org. Electron. 2019, 71, 296-302. https://doi.org/10.1016/j.orgel.2019.05.041.

(164) Lu, H.; Chen, X.; Anthony, J. E.; Johnson, J. C.; Beard, M. C. Sensitizing Singlet Fission with Perovskite Nanocrystals. J. Am. Chem. Soc. 2019, 141 (12), 4919-4927. https://doi.org/10.1021/jacs.8b13562.

(165) Medina, D. P.; Papadopoulos, I.; Lavarda, G.; Gotfredsen, H.; Rami, P. R.; Tykwinski, R. R.; Rodríguez-Morgade, M. S.; Guldi, D. M.; Torres, T. Light-Harvesting Porphyrazines to Enable Intramolecular Singlet Fission. Nanoscale 2019, 11 (46), 22286-22292. https://doi.org/10.1039/c9nr08161e.

(166) Daiber, B.; Pujari, S. P.; Verboom, S.; Luxembourg, S. L.; Tabernig, S. W.; Futscher, M. H.; Lee, J.; Zuilhof, H.; Ehrler, B. A Method to Detect Triplet Exciton Transfer from Singlet Fission Materials into Silicon Solar Cells: Comparing Different Surface Treatments. J. Chem. Phys. 2020, 
152 (11), 114201. https://doi.org/10.1063/1.5139486.

(167) Guo, D.; Ma, L.; Zhou, Z.; Lin, D.; Wang, C.; Zhao, X.; Zhang, F.; Zhang, J.; Nie, Z. Charge Transfer Dynamics in a Singlet Fission Organic Molecule and Organometal Perovskite Bilayer Structure. J. Mater. Chem. A 2020, 8 (11), 5572-5579. https://doi.org/10.1039/c9ta11022d.

(168) Ryasnyanskiy, A.; Biaggio, I. Triplet Exciton Dynamics in Rubrene Single Crystals. Phys. Rev. B Condens. Matter Mater. Phys. 2011, 84 (19). https://doi.org/10.1103/PhysRevB.84.193203.

(169) Reineke, S.; Baldo, M. A. Room Temperature Triplet State Spectroscopy of Organic Semiconductors. Sci. Rep. 2014, 4 (1). https://doi.org/10.1038/srep03797.

(170) Perkinson, C. F.; Tabor, D. P.; Einzinger, M.; Sheberla, D.; Utzat, H.; Lin, T. A.; Congreve, D. N.; Bawendi, M. G.; Aspuru-Guzik, A.; Baldo, M. A. Discovery of Blue Singlet Exciton Fission Molecules via a High-Throughput Virtual Screening and Experimental Approach. J. Chem. Phys. 2019, 151 (12), 121102. https://doi.org/10.1063/1.5114789.

(171) Wong, C. Y.; Penwell, S. B.; Cotts, B. L.; Noriega, R.; Wu, H.; Ginsberg, N. S. Revealing Exciton Dynamics in a Small-Molecule Organic Semiconducting Film with Subdomain Transient Absorption Microscopy. J. Phys. Chem. C 2013, 117 (42), 22111-22122. https://doi.org/10.1021/jp407645k.

(172) Wan, Y.; Guo, Z.; Zhu, T.; Yan, S.; Johnson, J.; Huang, L. Cooperative Singlet and Triplet Exciton Transport in Tetracene Crystals Visualized by Ultrafast Microscopy. Nat. Chem. 2015, 7 (10), 785-792. https://doi.org/10.1038/nchem.2348.

(173) Zhu, T.; Wan, Y.; Guo, Z.; Johnson, J.; Huang, L. Two Birds with One Stone: Tailoring Singlet Fission for Both Triplet Yield and Exciton Diffusion Length. Adv. Mater. 2016, 28 (34), 75397547. https://doi.org/10.1002/adma.201600968.

(174) Wan, Y.; Wiederrecht, G. P.; Schaller, R. D.; Johnson, J. C.; Huang, L. Transport of SpinEntangled Triplet Excitons Generated by Singlet Fission. J. Phys. Chem. Lett. 2018, 9 (23), 67316738. https://doi.org/10.1021/acs.jpclett.8b02944.

(175) Zhu, T.; Huang, L. Exciton Transport in Singlet Fission Materials: A New Hare and Tortoise Story. Journal of Physical Chemistry Letters. 2018, 9 (22), 6502-6510. https://doi.org/10.1021/acs.jpclett.8b02181.

(176) Jones, A. C.; Kearns, N. M.; Ho, J. J.; Flach, J. T.; Zanni, M. T. Impact of Non-Equilibrium Molecular Packings on Singlet Fission in Microcrystals Observed Using 2D White-Light Microscopy. Nat. Chem. 2020, 12 (1), 40-47. https://doi.org/10.1038/s41557-019-0368-9.

(177) Kwang, S. Y.; Frontiera, R. R. Spatially Offset Femtosecond Stimulated Raman Spectroscopy: Observing Exciton Transport through a Vibrational Lens. J. Phys. Chem. Lett. 2020, 11 (11), 4337-4344. https://doi.org/10.1021/acs.jpclett.0c01114.

(178) Reusswig, P. D.; Congreve, D. N.; Thompson, N. J.; Baldo, M. A. Enhanced External Quantum Efficiency in an Organic Photovoltaic Cell via Singlet Fission Exciton Sensitizer. Appl. Phys. Lett. 2012, 101 (11), 113304. https://doi.org/10.1063/1.4752445.

(179) Thompson, N. J.; Hontz, E.; Congreve, D. N.; Bahlke, M. E.; Reineke, S.; Van Voorhis, T.; Baldo, M. A. Nanostructured Singlet Fission Photovoltaics Subject to Triplet-Charge Annihilation. Adv. Mater. 2014, 26 (9), 1366-1371. https://doi.org/10.1002/adma.201304588.

(180) Zhang, Y.; Lei, Y.; Zhang, Q.; Xiong, Z. Thermally Activated Singlet Exciton Fission Observed in Rubrene Doped Organic Films. Org. Electron. 2014, 15 (2), 577-581. 
https://doi.org/10.1016/j.orgel.2013.12.010.

(181) Wu, T. C.; Thompson, N. J.; Congreve, D. N.; Hontz, E.; Yost, S. R.; Van Voorhis, T.; Baldo, M. A. Singlet Fission Efficiency in Tetracene-Based Organic Solar Cells. Appl. Phys. Lett. 2014, 104 (19), 193901. https://doi.org/10.1063/1.4876600.

(182) Bayliss, S. L.; Chepelianskii, A. D.; Sepe, A.; Walker, B. J.; Ehrler, B.; Bruzek, M. J.; Anthony, J. E.; Greenham, N. C. Geminate and Nongeminate Recombination of Triplet Excitons Formed by Singlet Fission. Phys. Rev. Lett. 2014, 112 (23). https://doi.org/10.1103/PhysRevLett.112.238701.

(183) Liu, H.; Jia, W. Y.; Zhang, Y.; Zhang, Q. M.; Lei, Y. L.; Lu, C. L.; Ling, Y. Z.; Xiong, Z. H. Tuning Magneto-Electroluminescence in Organic Light Emitting Diodes by Controlling the Competition between Singlet Fission and Triplet Fusion. Synth. Met. 2014, 198, 6-9. https://doi.org/10.1016/j.synthmet.2014.10.002.

(184) Qiao, X.; Luan, L.; Liu, Y.; Yu, Z.; Hu, B. Inter-Triplet Spin-Spin Interaction Effects on InterConversion between Different Spin States in Intermediate Triplet-Triplet Pairs towards Singlet Fission. Org. Electron. 2014, 15 (10), 2168-2172. https://doi.org/10.1016/j.orgel.2014.06.015.

(185) Tabachnyk, M.; Ehrler, B.; Gélinas, S.; Böhm, M. L.; Walker, B. J.; Musselman, K. P.; Greenham, N. C.; Friend, R. H.; Rao, A. Resonant Energy Transfer of Triplet Excitons from Pentacene to PbSe Nanocrystals. Nat. Mater. 2014, 13 (11), 1033-1038. https://doi.org/10.1038/nmat4093.

(186) Yang, L.; Tabachnyk, M.; Bayliss, S. L.; Böhm, M. L.; Broch, K.; Greenham, N. C.; Friend, R. H.; Ehrler, B. Solution-Processable Singlet Fission Photovoltaic Devices. Nano Lett. 2015, 15 (1), 354-358. https://doi.org/10.1021/nl503650a.

(187) Wakasa, M.; Kaise, M.; Yago, T.; Katoh, R.; Wakikawa, Y.; Ikoma, T. What Can Be Learned from Magnetic Field Effects on Singlet Fission: Role of Exchange Interaction in Excited Triplet Pairs. J. Phys. Chem. C 2015, 119 (46), 25840-25844. https://doi.org/10.1021/acs.jpcc.5b10176.

(188) Tisdale, J. T.; Li, M.; Hsiao, Y. C.; Wu, T.; Hu, B. Magneto-Optical Behaviors at a 2-D Ferromagnetic/Organic Semiconductor Interface for Singlet Fission. Org. Electron. 2017, 45, 169173. https://doi.org/10.1016/j.orgel.2017.03.011.

(189) Yonemura, H.; Futaoka, Y.; Taniguchi, T.; Sakaguchi, H.; Yamada, S. Effect of Silver Nanoparticle on Singlet Exciton Fission in Rubrene Films. Mol. Cryst. Liq. Cryst. 2017, 654 (1), 209-213. https://doi.org/10.1080/15421406.2017.1358046.

(190) Hodges, M. P. P.; Grell, M.; Morley, N. A.; Allwood, D. A. Wide Field Magnetic Luminescence Imaging. Adv. Funct. Mater. 2017, 27 (31), 1606613. https://doi.org/10.1002/adfm.201606613.

(191) Wakasa, M.; Yago, T.; Sonoda, Y.; Katoh, R. Structure and Dynamics of Triplet-Exciton Pairs Generated from Singlet Fission Studied via Magnetic Field Effects. Commun. Chem. 2018, 1 (1). https://doi.org/10.1038/s42004-018-0008-0.

(192) Cheng, C. Y.; Chitraningrum, N.; Chen, X. M.; Wen, T. C.; Guo, T. F. Magnetic Field Effect of the Singlet Fission Reaction in Tetracene-Based Diodes. Org. Electron. 2018, 56, 11-15. https://doi.org/10.1016/j.orgel.2018.01.036.

(193) Bayliss, S. L.; Weiss, L. R.; Mitioglu, A.; Galkowski, K.; Yang, Z.; Yunusova, K.; Surrente, A.; Thorley, K. J.; Behrends, J.; Bittl, R.; et al. Site-Selective Measurement of Coupled Spin Pairs in an Organic Semiconductor. Proc. Natl. Acad. Sci. U. S. A. 2018, 115 (20), 5077-5082. https://doi.org/10.1073/pnas.1718868115.

(194) Ishikawa, K.; Yago, T.; Wakasa, M. Exploring the Structure of an Exchange-Coupled Triplet Pair 
Generated by Singlet Fission in Crystalline Diphenylhexatriene: Anisotropic Magnetic Field Effects on Fluorescence in High Fields. J. Phys. Chem. C 2018, 122 (39), 22264-22272. https://doi.org/10.1021/acs.jpcc.8b06026.

(195) Tang, X.; Hu, Y.; Jia, W.; Pan, R.; Deng, J.; Deng, J.; He, Z.; Xiong, Z. Intersystem Crossing and Triplet Fusion in Singlet-Fission-Dominated Rubrene-Based OLEDs under High Bias Current. ACS Appl. Mater. Interfaces 2018, 10 (2), 1948-1956. https://doi.org/10.1021/acsami.7b17695.

(196) Zhu, H.; Jia, W.; Chen, L.; Tang, X.; Hu, Y.; Pan, R.; Deng, J.; Xiong, Z. Trap-Induced Conversion from Singlet Fission to Intersystem Crossing: Via in Situ Heating of Rubrene-Based Organic Light-Emitting Diodes. J. Mater. Chem. C 2019, 7 (3), 553-557. https://doi.org/10.1039/c8tc05677c.

(197) Yonemura, H.; Aira, D.; Asakura, N.; Ezoe, K.; Sakaguchi, H. Effect of Silver Nanoplate on Singlet Exciton Fission in Rubrene Polymer-Composite Films. Jpn. J. Appl. Phys. 2020, 59, SDDB03. https://doi.org/10.7567/1347-4065/ab51cc. 